\title{
SISTEMI GESTUALI TECNICI E LINGUAGGI DEI SEGNI COME ESEMPI DELLA COMUNICAZIONE GESTUALE ALTERNATIVA
}

\begin{abstract}
Szarota Beata, Sistemi gestuali tecnici e linguaggi dei segni come esempi della comunicazione gestuale alternativa [Gesture technical systems and sign languages as an example of alternative gesture communication]. Studia Romanica Posnaniensia, Adam Mickiewicz University Press, Poznań, vol. XXXVI: 2009, pp. 117-125. ISBN 978-83-232-2035-0. ISSN 0137-2475.
\end{abstract}

In the article we present short profiles and analysis of two gesture systems: the technical one - on the basis of conventional signs used by scuba-divers - and the Italian sign language in respect of signs of other languages. These are systems alternative to the gestures used in each verbal utterance and they show a lot of concurrence with language itself.

La gestualità è un concetto abbastanza ampio che comprende molti elementi di diversa natura. Principalmente possiamo distinguere due tipi di linguaggi gestuali:

- quelli che accompagnano e sono concomitanti alla comunicazione orale, in quanto da essa dipendono, usati da tutti gli interlocutori durante la conversazione;

- quelli ,alternativi" ${ }^{\prime}$, indipendenti dal linguaggio verbale che costituiscono i sistemi codificati e che servono a sostituire il messaggio verbale.

Nel nostro articolo faremo una breve presentazione ed analisi di due sistemi nonverbali alternativi, usati da alcuni gruppi professionali o/e dai sordomuti e che si oppongono alla gestualità intesa come i movimenti e le posture coinvolti nell'atto comunicativo allo scopo di sostenerlo, rafforzarlo, completarlo, accentuarlo oppure di rappresentare i schemi di pensiero che accompagnano in modo più o meno intenso, cosciente e intenzionato ogni enuncio verbale.

I sistemi gestuali indipedenti dal linguaggio verbale possono essere distinti in due tipi:

\footnotetext{
${ }^{1}$ Questa nozione è stata introdotta da Ricci Bitti (1988: 26).
} 


\section{SISTEMI GESTUALI „TECNICl”2}

Sono quei sistemi artificiali, convezionali, creati da diversi gruppi professionali (subacquei, pompieri, poliziotti, arbitri di gare sportive, operatori di borsa, oratori ecc.) o anche da gruppi etnici (sistemi di segni usati dalle diverse tribù p. es. indiane). Abbiamo qui a che fare con sistemi codificati chiusi, la cui conoscenza si limita solo a determinati gruppi professionali, cioè i gesti utilizzati dai subacquei sono compresi e usati dai rappresentanti solo di questo gruppo professionale; con i sistemi codificati semichiusi, la cui conoscenza al di là di un determinato gruppo professionale non è obbligatoria, tuttavia aiuta molto nella comprensione il contesto situazionale, come in caso di segni ufficiali, usati dagli arbitri e sportivi: i tifosi non devono conoscere questo sistema di segni, invece la loro conoscenza permette di capire meglio il gioco sul campo. Ci sono anche sistemi codificati aperti, che sono in realtà usati da persone che svolgono una determinata professione, invece quelli che devono essere correttamente decodificati dalla restante società, come per esempio il gesticolare del poliziotto che dirige il traffico al centro dell'incrocio.

Questi sistemi sono gruppi di una stretta quantità definita di segni, e così per esempio i subacquei usano $23 \mathrm{segni}^{3}$ indispensabili per capirsi sott'acqua, nella pallavolo gli arbitri utilizzano $30 \mathrm{segni}^{4}$, invece nel calcio ce ne sono quindici ${ }^{5}$.

Nei sistemi di gesti tecnici si distiguono tanto i segni arbitrari quanto quelli motivati poiché i gesti entrono in tre tipi di relazione:

a) causale-consecutiva, si tratta di gesti deittici che compaiono per esempio nella comunicazione dei sommozzatori: il gesto mostra una persona o indica col dito un determinato oggetto o direzione;

b) di somiglianza - riguarda i gesti iconici che rappresentano un elemento prototipo di un oggetto o di un'attività, a cui conducono. Per esempio: nella pallacanestro lo slancio al lato dell'arbitro con la mano destra piegata sul gomito indica che il giocatore ha spinto troppo in avanti il gomito nella gara; ugualmente nel hokey colpire all'altezza del petto, il gomito di un braccio con la mano aperta dell'altra mano indica attaccare l'avversario, ed è perciò una sorta di affronto. Fra i subacquei l'esecuzione di movimenti ondeggianti con l'indice e il medio tesi indica che il sommozzatore ha capogiri o fare cerchi con le mani a pugno significa ordinare di allacciare qualcosa;

c) simbolica ${ }^{6}$. È un tipo di relazione più spesso che compare nel sistema di gesti tecnici, poiché sono in maggioranza gesti arbitrari, come per esempio nel

${ }^{2}$ V. Dziechcińska (1996), Jarząbek (2002), Morris (1987), Ożdżyński (1979), Pyżo (1968), Ricci Bitti (1988).

${ }^{3}$ Secondo CMAS.

${ }^{4}$ Secondo FIVB.

${ }^{5}$ Secondo FIFA.

${ }^{6}$ Più diffusamente scrive della relazione tra segno e oggetto fra l'altro U. Żegleń (2000: 41-42). Indica tre tipi di relazioni: a) relazione causale-consecutiva, b) di somiglianza, c) di espressione 
calcio mostrare il cartellino rosso o giallo, per subacquei il gesto di negazione o di ordine per aprire la riserva.

Alcuni dei gesti tecnici compaiono parallelamente nella comunicazione normale sia come forme sinonimiche (gesti deittici dei subacquei, il loro gesto di esonero della velocità nell'attività effettuata, il gesto di $\mathrm{OK})$ o come gesti di false somiglianze semantiche (omonimi gestuali, falsi amici gestuali), come ad esempio: il pollice alto o il pollice verso nel sistema gestuale dei subacquei significano l'ordine di nuotare verso l'alto o verso il basso; il gesto di toccare verticalmente la gola con la mano aperta non è una forma di minaccia, ma il segno di mancanza d'aria oppure il gesto di aver delle vertigini può esser interpretato da un italiano che ignora il suo significato, come segno di morte.

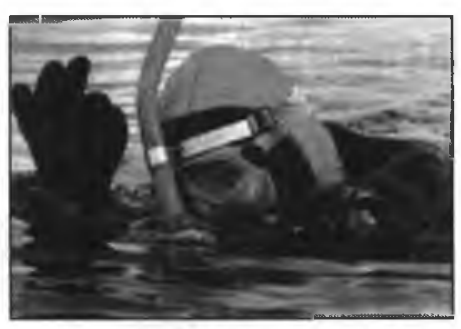

Il gesto di OK è usato e codificato anche nella comunicazione dei subacquei

Praticamente i gesti ,tecnici” compaiono separati, le loro unità hanno un valore come unità isolate, senza far riferimento agli altri segni gestuali e utilizzano l'espressività di una determinata, generale idea e non invece di singole lettere o parole.

Un'eccezione interessante è costituita dai gesti ibri$d i^{7}$, in realtà anche se raramente e pur presenti nella comunicazione: sono quelli che combinano due gesti distinti, con origini diverse, in un'unica azione (Morris 1987: 43). Naturalmente possiamo fare una serie di gesti, uno dopo l'altro, ma in caso degli ibridi abbiamo un esempio del fondere due azioni distinte in una nuova ,uni-tà doppia", come fanno per esempio gli indiani del Nord America che indicano „la bellezza": combinando il gesto „guardare" (ossia fissarsi il palmo della mano come se imitassero lo specchio) e il segno di „buono” (porre la mano a taglio sul petto con il palmo rivolto in giù) (1987: 43).

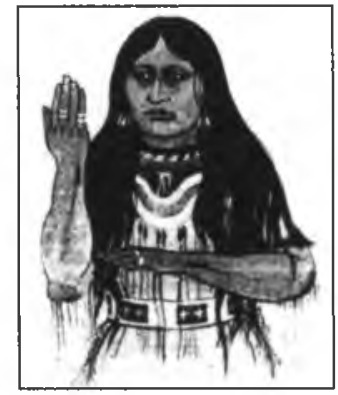

Esempio di un gesto ibrido Morris (1987: 43)

I sistemi dei segni tecnici possono essere anche aiutati con l'uso di oggetti. Essi sono una forma di ,prolungamento del lavoro del braccio”, per questo aumenta il loro campo di visibilità e causano la presa in considerazione degli altri partecipanti alla comunicazione. Un esempio è il sistema di segni che indicano il contenuto con l'aiuto della bandierina. Si utilizza anche la torcia (i segni dati di notte dai subacquei), fischietti e palette (usati dai poliziotti).

psichica. Però nel caso sopraccitato di tipi di gesti tecnici, bisogna piuttosto parlare di relazione simbolica, poiché questi segni sono usati sono nell'ambito della cassa toracica e delle mani, ma non considerano la mimica del viso.

${ }^{7}$ La nozione dei gesti ibridi compare per la prima volta da Efron (1941). 

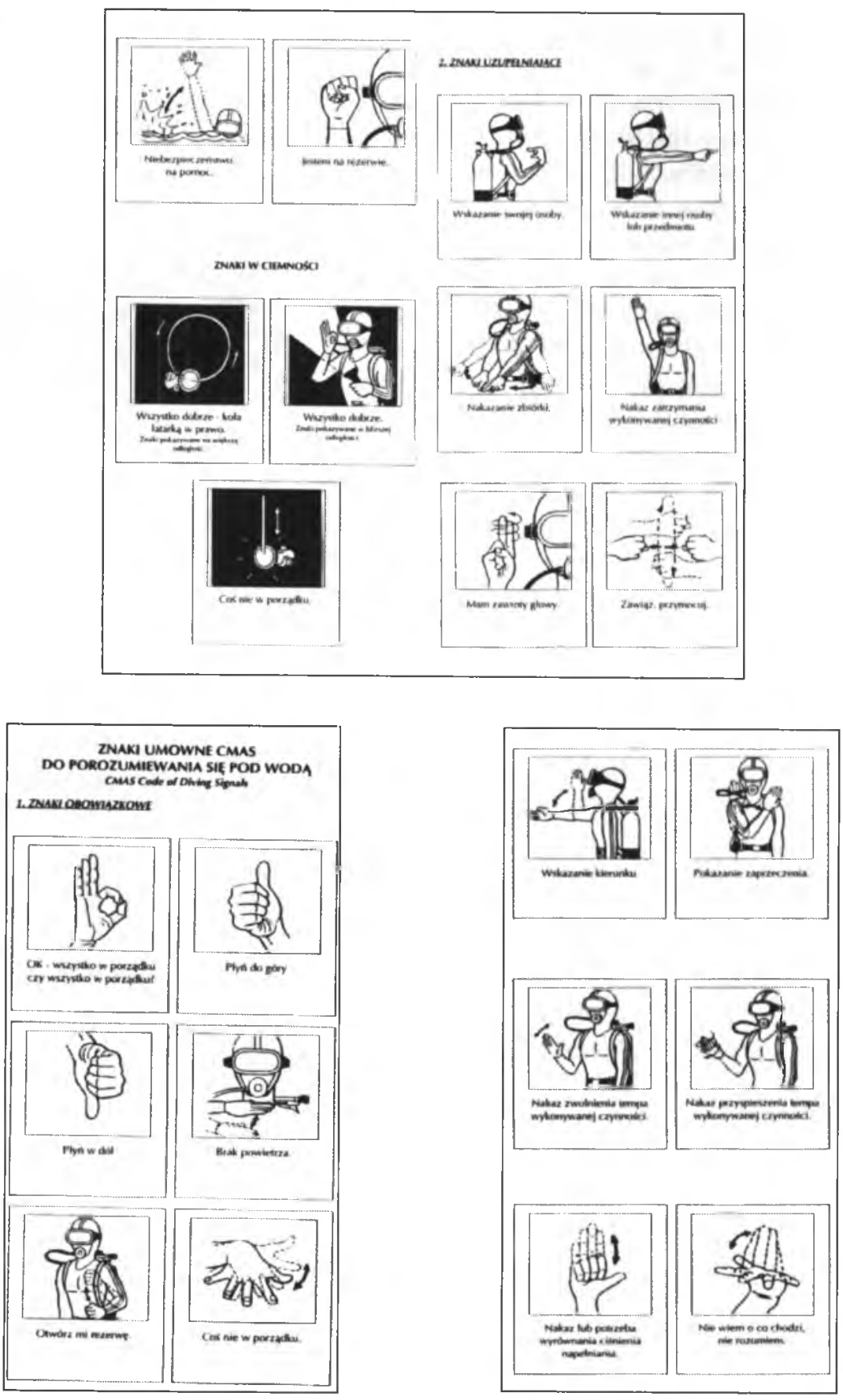

Segni gestici CMAS usati dai subacquei

Fonte: Ksiqzeczka ptetwonurka KDP, 2005 
Riassumendo possiamo affermare che tutti i segni tecnici sono segni convenzionali, creati per facilitare comunicazioni veloci, in situazioni quando non è possibile usare il linguaggio verbale sia in convenzioni culturali (la regola di tacere in un monastero) che fisiche:

- una grande lontananza tra i locutori non permette una comunicazione orale;

- $\mathrm{i}$ locutori sono in movimento continuo (gli sportivi) e il segno gestuale viene compreso più velocemente dalla parola (Jarząbek 2002: 154);

- Rumore o condizioni atmosferiche, ambientali (la comunicazione sott'acqua) escludono altri modi di comunicare.

Il sistema contrattuale dei segni gestuali è più economico e preciso e costituisce „lo scambio di brevi espressioni verbali - di frasi imperative, e di equivalenti frasi indicative e singole parole" (Jarząbek 2002: 153).

\section{LINGUAGGI DEI SEGNI}

I sistemi usati dai sordomuti sono costituiti dal linguaggio dei segni.

I sistemi dei segni gestuali sono definiti come il linguaggio verbale dal carattere non vocale (Caselli, Pagliari Rampelli 1987, Poggi 1987, Radutzky 1988, Romeo 2002, Szczepankowski 2002).

Non è una lingua universale: qualunque comunità di sordi sviluppa e utilizza la propria lingua di segni, e anzi all'interno di uno stesso paese possono esistere diverse varietà di una stessa lingua di segni (dialetti). Infatti esistono grandi somiglianze per esempio tra la LIS $^{8}$, lingua dei segni russa e quella svizzera, che erano molto influenzate da quella francese, anche se in generale sono lingue diverse. La cosa interessante è anche che se ci sono perfino somiglianze tra i linguaggi dei segni, queste non sono analoghe (lessicali, morfosintattiche) alle lingue verbali, che appartengono alla stessa famiglia linguistica, ad esempio: la lingua dei segni britannica (BSL) non assomiglia affatto a quella americana (v. Radutzky 1988: 87).

I linguaggi dei segni fanno parte di un sistema comunicativo analogico-digitale (cfr. Watzlawick e altri 1981) poiché i segni rivelano un carattere paradossale, vale a dire sono contemporaneamente motivati e arbitrari (Klima, Bellugi 1980, in: Pavelin 2002: 24).

I segni dei sordi sono in maggior parte iconici poiché rappresentano in modo metonimico le caratteristiche essenziali fisiche, le caratteristiche prototipe dell'oggetto trattato. E così per esempio in modo figurato sono illustrati i segni che riguardano alcuni animali indicando con le braccia la forma delle loro corna e orecchie (nella LIS - asino, cavallo, coniglio) ugualmente la grandezza (altezza) è allo stesso modo espressa; le idee di alcuni verbi sono rappresentati mimando l'azione determinata (aprire, chiudere, scrivere, tagliare, pesare, cadere) oppure si

\footnotetext{
${ }^{8}$ Lingua Italiana dei Segni.
} 
presenta in questo modo gli oggetti di diverso tipo, imitando le loro forme, come per esempio il telefono o gli occhiali.

Tuttavia questa iconicità non è sempre abbastanza evidente e chiara. Le ricerche di E. Radutzky hanno indicato che di 100 segni apparentemente iconici, gli udenti che non ne conoscono il significato riescono a indovinarlo solo nel 10-15\% dei casi (Poggi 1987: 108).

Secondo Poggi (1987: 108) questo testimonia la presenza di arbitrarietà in sistemi di comunicazione, che pur naturalmente portano all'iconicità, e ciò si spiega in due modi:

1) in modo sincronico: nonostante iconici i segni rappresentino i tratti tipici dell'oggetto di cui si parla ma si può prendere in considerazione i tratti diversi, ad esempio: il segno per "gatto" nell'ASL (American Sign Language) consiste nel lisciarsi un imaginario baffo, e nella LIS (Lingua Italiana dei Segni) nel fingere di graffiarsi una mano (esempio di Poggi 1987: 108). Si vede che gli esempi presentano i vari aspetti dello stesso referente (gatto - quello che ha i baffi, gatto - quello che graffia) quindi selezionarne solo un aspetto è arbitrario;

2) in modo diacronico: si osserva la tendenza dei segni a diventare più astratti e stilizzati, fino a perdere l'originaria iconicità. Inoltre, alcui segni originariamente iconici, hanno perso la loro trasparenza. ad esempio: il segno per "sabato" nella LIS di Roma in cui la mano destra fa le corna battendo sul petto. È qui oscurato l'orignario legame fra segnale e significato che passava attraverso associazioni metonimiche „sabato - ebrei - diavolo - corna” (esempio di Poggi 1987: 108).

Esistono anche segni motivati di carattere deittico. Si usano per indicare il soggetto dell'enunciato (di se stesso, dell'altro interlocutore).

$\mathrm{Ci}$ sono anche segni abitrari e riguardano idee astratte, cose non materiali, lo scandire del tempo, le caratteristiche di carattere, le istituzioni, alcuni paesi, colori, cifre ecc.

Anche qui possiamo osservare i casi della dualità paradossale di segni che sono ugualmente motivati e arbitrari: c'è la dualità deittico-simbolica. Si tratta dei segni che esprimono alcuni aspetti del tempo e dello spazio, cioè, ad esempio: „domani”, „ieri”, „l'altro ieri”, „qui”, ,adesso - oggi”, di cui la configurazione delle dita (forma) sono tipiche per i segni deittici (muovere l'indice teso mentre le altre dita restano chiuse). Inoltre compare qui anche un modo egocentrico di concepire e rappresentare il tempo e lo spazio, questo è una delle caratteristiche principale della regola di iconocità nella lingua. Il corpo umano è il punto di partenza a definire il tempo e lo spazio, e così l',,idea" del tempo attuale e di ,qui” è situata proprio vicino al proprio corpo e cioè ai piedi; il tempo passato è dietro (le spalle) mentre il futuro davanti a noi. È d'accordo con il nostro modo pienamente arbitrario $^{9}$ di concepire la spaziotemporalità. Oltre alla concezione della metafora orien-

${ }^{9}$ Gli studi linguistici condotti tra gli indiani dell'America Latina dimostrano che il tempo concepito come metafora d'orientamento AVANTI-DIETRO è totalmente arbitraria e convenzionale, 
tativa del tempo (AVANTI - DIETRO) (v. Lakoff 1988), abbiamo qui anche l'idea del TEMPO visto come PUNTO (presente) e LINEA (dalla parte destra - futuro, dalla sinistra - passato). Gli esempi concreti sotto trattano solo la Lingua Italiana dei Segni, invece i principi generali riguardano l'insieme dei segni dei sordi.
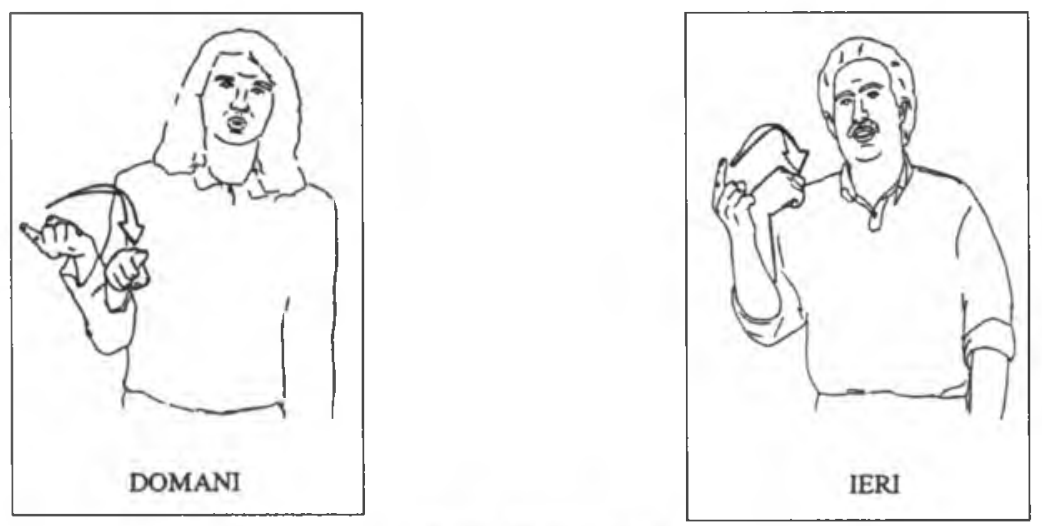

Fonte: Romeo 2002

Bisogna anche riconoscere che si osservano delle convergenze fra $i$ segni dei sordi e $i$ gesti degli udenti. Nello stesso modo gli Italiani udenti (specialmente i meridionali) esprimono il tempo (,,ieri”, „domani”), lo spazio (,qui”), alcuni tratti del carattere (,furberia”), aggettivi (,duro”, „buono”), le azioni (,telefonare”, „dormire”, „invitare ad avvicinarsi”), qualche forma di misura o di quantità (,metà", ,tanto") ma anche gli altri.
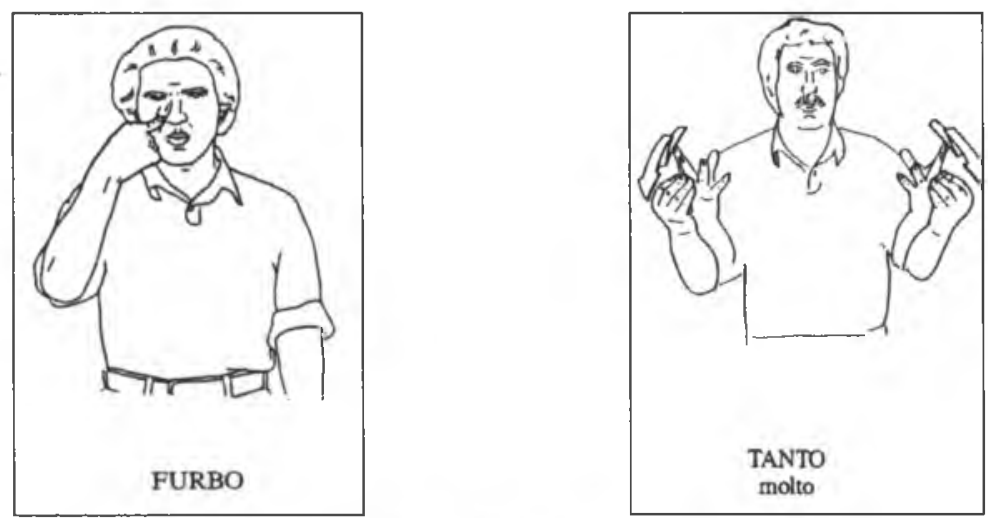

Fonte: Romeo 2002

poiché in queste societâ, il passato è davanti (come già è risaputo e visto), invece il futuro è indietro, come qualcosa che non conosciamo, quindi non possiamo vedere e va oltre la portata del nostro sguardo (v. Calbris, Montredon 1986, Calbris, Porcher 1989, Montredon 1998). 
Una caratteristica successiva delle lingue dei segni è la possibilità di considerare anche gli aspetti grammaticali. Certo queste lingue sono molto più povere di elementi sintattico-morfologici, ma le lingue di segni esprimono non solo idee isolate ma anche frasi. „In realtà, la LIS ad esempio, come le altre lingue di segni, adotta meccanismi particolari per stabilire le relazioni fra gli elementi delle frasi e per esprimere gli aspetti morfologici. Questi meccanismi sono:

- l'uso dello spazio

- la modulazione del movimento" (Caselli, Pagliari Rampelli 1987: 9).

Ad esempio il plurale di un nome viene marcato attraverso una ripetizione del movimento del segno e un'estensione dello spazio occupato dal segno (cfr. Caselli, Pagliari Rampelli 1987, Radutzky 1988).

Contrariamente alla lingua italiana vocale, la LIS è una lingua di posizione e per questo, ad esempio, le preposizioni come „di”, „con” sono espresse attraverso una struttura adeguata di posizione di elementi della frase, e così la frase del tipo: "Il tavolo è di legno” nella LIS sembra: „Tavolo legno”, invece „Parto con l'aereo” è „Io aereo (partire)” (esempi di Caselli, Pagliari Rampelli 1987: 12).

Un'importante funzione distintiva la adempie qui anche la mimica. In alcuni segni la modificazione dell'espressione facciale fa parte integrante del segno stesso e riempie il ruolo semantico come ad esempio negli aggettivi „amaro”, „,bello” o nel termine ,violenza".

La mimica corrispondente serve a distinguere le diverse modalità sintattiche, come le frasi dichiarative (assenza di un segnale specifico), le domande sì-no (innalzamento delle sopracciglia), altre domande (corrugamento della fronte, alzamento delle sopracciglia), frase imperativa (squardo fisso sul ricevente), frase condizionale (testa reclinata), negazione (suotere la testa, corrugamento della fronte), affermazione (cenno in avanti della testa), topicalizzazione (testa inclinata e le ciglia alzate), frase relativa (alzamento delle ciglia, delle guance, del labbro e piegamento all'indietro della testa) (Radutzky 1988: 105-106).

Le lingue dei segni costitiscono ampi e aperti sistemi lessicali che danno la possibilità di creare sempre nuovi messaggi linguistici. Ci sono forme sinonimiche (ordine „basta”, verbo "farsi il bagno") e anche omonimi (il gesto che riguarda ugualmente „circo" e „clown”).

Per quanto riguarda la forma ne possiamo dividere in tre gruppi:

- le figure semplici, monofasiche, dove succede solo un movimento nello spazio (p. es. alto-basso) senza un cambiamento di necessità della configurazione della mano;

- le figure doppie che specificano la posizione iniziale e quella finale;

- e la forma estrema: i gesti ibridi, che costituiscono il legame di due diversi segni separati nella totalità semantica.

I sistemi dei segni dei sordi sono una specie della lingua non vocale, in cui individuiamo somiglianze in riguardo a forme e significati verso un sistema gestuale di udenti. Ma in caso della lingua dei segni abbiamo a che fare con la 
lingua, che come ogni lingua include anche elementi grammaticali ed è un sistema illimitato e autonomo comunicativo; significa che permette ai suoi utenti di esprimere tutti i contenuti voluti.

Riasumendo, possiamo constatare che i sistemi tecnici e linguaggi dei segni costituiscono i sistemi codificati, convenzionali, limitati dal punto di vista quantitativo che rivelano sia il carattere motivato, cioè somigliano al gesto, sia il carattere arbitrario e per questo si avvicinano alla parola.

\section{BIBLIOGRAFIA}

Calbris G., Montredon J. (1986), Des gestes et des mots pour le dire, Paris : CLĖ International.

Calbris G., Porcher L. (1989), Geste et communication, Paris : Hatier.

Caselli C., Pagliari Rampelli L. (1987), LIS-Lingua Italiana dei Segni, Italiano e oltre, nr 1 (gennaio febbraio), La Nuova Casa Editrice, p. 8-13.

Dziechcińska H. (1996), Ciato, strój, gest w czasach renesansu i baroku, Warszawa : Wydawnictwo Naukowe Semper.

Efron D. (1941, rist.1974), Gesto, razza e cultura, Milano : Bompiani.

Jarząbek K. (2002), Mowa ciała ludzkiego, in: Mowa ciata i jej funkcje w kulturze, red. M. Jasińska, J. Kuć, Siedlce : Wydawnictwo Akademii Podlaskiej, p. 5-13.

Lakoff G., Johnson M. (1988), Metafory w naszym życiu. Warszawa : PWN.

Montredon J. (1998), Comment la gestuelle a joué parfois identiquement, parfois différemment pour figurer à partir des positions et d'oppositions spatiales universelles (droite/gauche, haut/bas, devant/derrière), des axes ou des sites temporels (passé, présent, futur), in: Oralité et gestualité, red. S. Santi, I. Guailtella, G. Konopczynski, Ch. Cav, Paris : l'Harmattan, p. 147-152.

Morris D. (1987), L'uomo e i suoi gesti: la comunicazione non-verbale nella specie umana, Milano: Arnoldo Mondadori Editore.

Ożdżyński J. (1979), Pozajęzykowe formy środowiskowego komunikowania, in: Mówione warianty w środowisku sportowym, Wrocław-Warszawa-Kraków-Gdańsk : Ossolineum.

Pavelin B. (2002), Le geste à la parole, Toulouse : Presses Universitaires du Mirail.

Poggi I. (1987), La comunicazione verbale e non verbale, in: Le parole nella testa : guida a un'educazione linguistica cognitivista, Bologna : Il Mulino, p. 99-127.

Pyżo W. (1968), Język gestów, Kultura fizyczna, nr 11, p. 518-520.

Radutzky E. (1988), La lingua dei segni dei sordi e la comunicazione "non verbale", in: Comunicazione e gestualitâ (red. P.E. Ricci Bitti), Milano : Franco Angeli, p. 86-107.

Ricci Bitti P.E. (1988), Comunicazione e gestualità, Milano : Franco Angeli.

Romeo O. (2002), Dizionario dei segni, Bologna : Zanichelli.

Szczepankowski B. (2002), Język migowy a mowa ciata, in: Mowa ciała i jej funkcje w kulturze (red. M. Jasińska, J. Kuć), Siedlce : Wydawnictwo Akademii Podlaskiej, p. 127-138.

Watzlawick P., Beavin J.H., Jackson D.D. (1972), Une logique de la communication, Paris : Éditions du Seuil.

Żegleń U.M. (2000), Wprowadzenie do semiotyki teoretycznej i semiotyki kultury, Toruń : Wydawnictwo Uniwersytetu Mikołaja Kopernika. 